B. B. Rokhman, orcid.org/0000-0002-1270-6102,

N. I. Dunayevska, orcid.org/0000-0003-3271-8204, V. G. Vyfatnuik, orcid.org/0000-0003-0771-2652,

I. V. Beztsennyi, orcid.org/0000-0001-6536-5121
Thermal Energy Technology Institute of the National Academy of Sciences of Ukraine, Kyiv, Ukraine, e-mail: brolene@yahoo.com

\title{
CO-FIRING OF GAS COAL DUST FINE PARTICLES AND SYNTHETIC PEAT GAS. \\ PART 1. SIMULATION OF PROCESSES OF STEAM-AIR GASIFICATION OF PEAT IN A FIXED BED AND COMBUSTION OF DUST AND GAS MIX IN A STREAM
}

Purpose. To build a model of solid fuel gasification in a fixed bed taking into account the velocity of particles, which allows obtaining detailed information about the technological process. To develop an optimal technological scheme of co-firing of pulverized coal particles and peat gas in the TPP-210A boiler. To investigate the process of a binary mix burning.

Methodology. The objects of research were pulverized coal of Ukrainian deposits and peat from Volyn region deposits. The developed model was used to calculate the peat gasification process. To study the process of burning a binary blend, ANSYS FLUENT package was used.

Findings. A model of solid fuel gasification in a fixed bed has been developed, which differs from the existing models by taking into account the particle velocity and its change. With the aid of this model the detailed information on temperature and concentration fields of the two-phase environment is received. The process of burning binary mix in a TPP-210A boiler is investigated.

Originality. It was found that in the time interval $2200<\tau<3200 \mathrm{~s}$ a stable stationary process of thermochemical processing of peat with $q_{4.1}=0.13-0.3 \%$ is formed. The profile of the dispersed phase velocity distribution resembles a mirror reflection of the profile of the particle diameter dependence.

Practical value. Using the constructed model, the process of thermochemical processing of $28939 \mathrm{~kg} / \mathrm{h}$ of raw peat in three reactors with a fixed bed at a pressure of $1.5 \mathrm{MPa}$ was numerically investigated. The composition of the generator gas at the reactor outlet was determined.

Keywords: fixed bed, coal, peat, thermal conductivity, steam-air gasification, conductive and radiative heat exchange

Introduction. Problem of finding renewable types of material sources and the technologies of their thermochemical processing for industry and power sector are extremely topical. And one of prioritized directions in this respect is the joint combustion of products of biomass gasification with coal in combustion chambers of steam boilers. The main advantage of solid fuel gasification under pressure, in contrast to the gasification at atmospheric pressure, is a reduction of the dimensions of the gas generator resulting from the higher speed of homogeneous and heterogeneous chemical reactions due to growth of the concentrations (proportionally to pressure) of gas components, which is very important for installing the gasifiers at existing power units.

Literature review. In [1], the biomass gasification process occurs in a circulating bubbling bed. The obtained generator gas together with a coke residue is fed to the discharge burners of the steam generator with a wet slag removal. The grinded coal transported by a hot air is supplied to the main burners of the boiler.

In this paper, a method is proposed for combustion of the binary mixture in the boiler of the TPP-210A steam generator with a wet slag removal 7(Fig. 1); the mixture consists of polydisperse dust coal particles and peat gas 6 , generated by thermochemical processing of Volyn peat in the fixed bed 18 in a steam-air mixture 14 enriched with oxygen, under pressure of 1.5 $\mathrm{MPa}$.

The process in the gas generator, based on the changes that peat undergoes at different stages of thermochemical processing, could be divided into four zones. The slag pillow (zone $I$ in Fig. 1) consists of ash particles formed as a result of com-

(C) Rokhman B. B., Dunayevska N. I., Vyfatnuik V. G., Beztsennyi I. V., 2021 bustion and gasification of the initial fuel. This zone protects the grate of the reactor 15 from overheating. Oxidizing zone II includes two areas. In the first, where most of the oxygen is intensively spent in a very narrow layer on the conversion of carbon and afterburning of gasification products $\mathrm{CO}$ and $\mathrm{H}_{2}$, the temperature of gas and that of peat particles reach maximum values. In the second zone (oxidation-reducing region of reacting), heat absorption in endothermic reactions $\mathrm{C}+\mathrm{CO}_{2}=$ $=2 \mathrm{CO}$ and $\mathrm{C}+\mathrm{H}_{2} \mathrm{O}=\mathrm{CO}+\mathrm{H}_{2}$ dominates over the heat release from exothermic reactions $\mathrm{C}+\mathrm{O}_{2}=\mathrm{CO}_{2}, \mathrm{C}+0.5 \mathrm{O}_{2}=\mathrm{CO}$, $\mathrm{CO}+0.5 \mathrm{O}_{2}=\mathrm{CO}_{2}$ and $\mathrm{H}_{2}+0.5 \mathrm{O}_{2}=\mathrm{H}_{2} \mathrm{O}$, resulting in a sharp drop in temperatures of the phases and in $\mathrm{O}_{2}$ concentration. The reduction zone III is more extended than the oxidizing region due to lower velocities of heterogeneous reducing reactions with a relatively low level of the discrete phase temperature and concentrations of gas components $\mathrm{CO}_{2}$ and $\mathrm{H}_{2} \mathrm{O}$. In the fourth zone, the dried particles of the peat are heated with the oxygen-free gas mix through the interphase convective heat transfer and the radiation-conductive heat transfer of the solid phase, which provides the yield of volatile substances that mixes with the gas environment entering from the third zone, thereby forming a generator gas at the outlet of the plant 11 . After passing the scrubber 12 , the generator gas 13 is directed to the cleaning chamber 17 , where it is cleaned from dust and tar, after which the tar burns in the combustion chamber 20 and the heat of combustion products is spent on evaporation of moisture from the raw peat 21 in a recuperative drying chamber 22. If needed, an increase in the temperature of the drying agent is possible by recirculation of the portion of gas through the pipeline 25 into the combustion chamber. The amount of recirculated generator gas is regulated by the valve 24 . The raw peat is supplied to the gateway bunker 33 , after which a pressure of 1.5 MPa is established due to the introduction of a gen- 


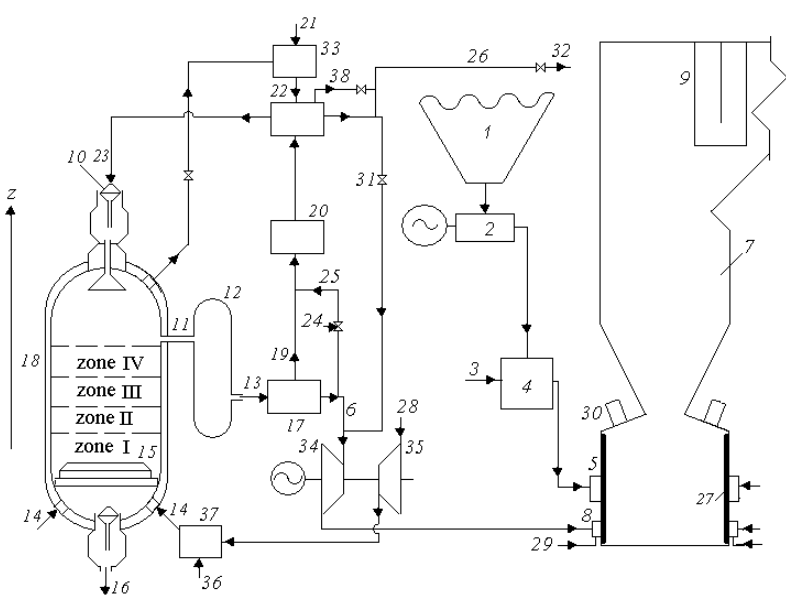

Fig. 1. Scheme of joint combustion in the furnace of TPP-210A boiler of gas coal and synthetic peat gas:

1 -dust bunker; 2 -dust feeder; 3 - transport agent; 4 - mixer; 5 - the main burners; 6 - synthetic peat gas; 7 - furnace; 8 - gas burners; 9-screens; 10 - flashing; 11 - the output of the generator gas; 12 - scrubber; 13 - raw gas outlet; 14 - steam-air mixture; 15 - grate; 16 - ash discharge; 17 - gas cleaning; 18 - gas generator; 19 - supply of tar to the combustion chamber; $20-$ combustion chamber; 21 - raw peat feeding; 22 - drying chamber; 23 - dried peat; 24 - control valve; 25 - supply of generator gas to the combustion chamber; 26 - the discharge of the raw peat moisture; 27 - liquid slag; 28 - air enriched with oxygen; 29 - air; 30 - discharge burners; 31 - damper; 32 -dumping of moisture outside the boiler; 33 - gateway bunker; 34 - gas turbine; $35-$ compressor; 36 - steam; 37 - mixing chamber; 38 - discharge of combustion products outside the boiler; I-slag pillow; II-oxidation zone; III - gasification zone; IV-pyrolysis zone

erator gas to the hopper from reactor 18 . The dried peat from the drying chamber 22 enters the gas generator. The coal dust from bunker 1 is fed into the mixer 4 dust feeder 2 , where it is mixed with a transport agent 3 , and directed into the main burner 5. Cleared from tar and dust, the synthetic peat gas 6 is mixed with evaporated moisture of raw fuel 26 and enters gas turbine 34 , wherein the excess pressure is reduced to atmospheric pressure, after which the generator gas is fed into gas burners 8 , located $455 \mathrm{~mm}$ from the bottom. The air necessary for combustion 29 is also directed there. In this case, generator peat gas contains $22.6 \% \mathrm{H}_{2} \mathrm{O}$, which reduces its calorific value and contributes to condensation of $\mathrm{H}_{2} \mathrm{O}$ in turbine 34. To eliminate these disadvantages, it is proposed to discharge the evaporated moisture 26 and spent drying agent 38 by-passing the boiler through the pipeline 32 . In this case, it is advisable to use a regenerative drying chamber 22 . In addition, in the cleaning chamber 17 it is necessary to install two recuperative heat exchangers. The first of them is intended to cool the peat gas, resulting in condensation of moisture. The second heat exchanger serves to transfer heat to the dried generator gas extracted in the first heat exchanger.

The gas turbine drives the compressor 35 in which the compression of air 28 enriched with oxygen occurs. The steamair mixture 14 is formed in a mixer chamber 37 , where steam 36 and air 28 are supplied. The composition and caloric value of the generator peat gas introduced into the reaction zone can be adjusted by changing the degree of enrichment of the reacting agent with oxygen and water vapor. Unlike the technology of combustion of binary mixtures in the furnaces with wet slag removal [1], where a similar scheme (Fig. 1) is realized, which is used in European countries, the major problem in organizing the process in the furnaces with wet slag removal 27 is to secure the steady leakage of slag through the tap-hole. This problem is solved with installation of gas burners 8 combusting synthetic gas. In the scheme of joint combustion of binary mixture in Fig. 1, the main elements are the gas generator 18 and the com- bustion chamber of the existing boiler TPP-210A 7. The TPP$210 \mathrm{~A}$ boiler is equipped with three gasifiers installed in series. Such allocation minimizes the plant space.

Unsolved aspects of the problem. In order to obtain detailed information about aerodynamic, thermal and physical and chemical parameters of steam-air gasification of solid fuel under pressure in a fixed bed and the required design characteristics of the plant, it is necessary to develop a mathematical twodimensional model based on the construction of a two-dimensional system of parabolic and hyperbolic equations of the reacting multiphase medium.

Purpose. To construct a solid fuel gasification model in a fixed bed, which differs from existing models by accounting the particle velocity and its changes, which allows obtaining detailed information about the technological process. To develop an optimal technological scheme of joint combustion of coal dust particles and peat gas in the boiler of TPP-210A. To investigate the process of binary mix combustion.

Methods. It is assumed that the process of steam-air gasification is quasi-stationary, gas mixture in the gas generator consists of oxygen, carbon dioxide and monoxide, methane, water vapor, hydrogen, nitrogen, acetylene and oxygen-containing compounds, the solid phase is represented by monodisperse particles of spherical shape, yield of volatile matter causes a decrease in the density of the solid phase at a constant diameter, and heterogeneous combustion and gasification reactions on the surface of the particles cause the decrease in their size at unchanged density, the porosity of the bed does not vary over the vertical axis $z$, the gas between the particles in the bed moves in the mode of ideal displacement, the stoichiometric scheme of the reactions includes four heterogeneous reactions

$$
\begin{gathered}
\mathrm{C}+\mathrm{O}_{2}=\mathrm{CO}_{2} \mathrm{C}+0.5 \mathrm{O}_{2}=\mathrm{CO} \quad \mathrm{C}+\mathrm{CO}_{2}=2 \mathrm{CO} \\
\mathrm{C}+\mathrm{H}_{2} \mathrm{O}=\mathrm{CO}+\mathrm{H}_{2}
\end{gathered}
$$

and five homogeneous reactions

$$
\begin{gathered}
\mathrm{CH}_{1.193} \mathrm{O}_{0.4083} \rightarrow 0.205 \mathrm{CO}+0.13 \mathrm{CO}_{2}+0.33266 \mathrm{CH}_{4}+ \\
+0.1625 \mathrm{C}_{2} \mathrm{H}_{2}+0.0256 \mathrm{H}_{2}+0.375 \mathrm{H}_{2} \mathrm{O} \\
\mathrm{CO}+0.5 \mathrm{O}_{2}=\mathrm{CO}_{2} \\
\mathrm{H}_{2}+0.5 \mathrm{O}_{2}=\mathrm{H}_{2} \mathrm{O} \\
\mathrm{CO}+\mathrm{H}_{2} \mathrm{O}=\mathrm{CO}_{2}+\mathrm{H}_{2} \\
\mathrm{CH}_{4}+\mathrm{H}_{2} \mathrm{O}=\mathrm{CO}+3 \mathrm{H}_{2}
\end{gathered}
$$

In the stoichiometric scheme of the reactions, the volatile components of the peat are represented by the oxygen-containing compound $\mathrm{CH}_{1.193} \mathrm{O}_{0.4083}$, which, when heated, is decomposed to gas components $\mathrm{CO}, \mathrm{CO}_{2}, \mathrm{CH}_{4}, \mathrm{C}_{2} \mathrm{H}_{2}, \mathrm{H}_{2}$ and $\mathrm{H}_{2} \mathrm{O}$ according to the first reaction (2).

The bed is heated up with a high-temperature gas mixture to a temperature of ignition of particles $\left(\sim 600^{\circ} \mathrm{C}\right)$ contained in a narrow area adjacent to the bottom. This moment is taken for the beginning of the countdown $\tau=0$. Then the heat supply is cut and the feeding starts of a steam-air mixture enriched with $\mathrm{O}_{2}$, with a temperature $t_{\mathrm{H}_{2} \mathrm{O}+\text { air }}=350-450{ }^{\circ} \mathrm{C}$.

Unlike in [2], where a non-stationary process of coal steam-oxygen gasification is studied with a singlefold (nonrestorable) loading of a solid phase on a grating lattice, here a quasi-stationary process is considered of peat gasification with a continuous feeding of the raw fuel and ash and slag discharge from the gasifier. As a result, the distribution of phases and concentrations of gas components vary weakly in time.

In existing methods of calculating the fixed bed [2], the velocity of the particles is $u_{\mathrm{p}}(\tau, z)=0$, while in the proposed model $u_{p}(\tau, z) \neq 0$. Moreover, in the oxidative and reducing zones the disperse phase velocity is changing $\partial u_{p} / \partial z \neq 0$ due to the decrease in diameter of particles as heterogeneous reactions (1) progress. This is the main distinction of the proposed 
model from existing models [3]. The differential equation describing the change in the peat particles velocity in the oxidizing and reducing zones can be obtained from the continuity equation for the disperse phase [4]

$$
\begin{gathered}
\frac{\partial \rho_{p}}{\partial \tau}+\frac{\partial\left(u_{p} \rho_{p}\right)}{\partial z}= \\
=-\left[\left(k_{\mathrm{C}+\mathrm{O}_{2}}+2 k_{\mathrm{C}+0.5 \mathrm{O}_{2}}\right) C_{\mathrm{O}_{2}}^{w}+k_{\mathrm{C}+\mathrm{CO}_{2}} C_{\mathrm{CO}_{2}}^{w}+k_{\mathrm{C}+\mathrm{H}_{2} \mathrm{O}} C_{\mathrm{H}_{2} \mathrm{O}}^{w}\right] \frac{6 \mu_{C}}{\delta} .
\end{gathered}
$$

Taking into account that $\rho_{p}=$ const in the considered zones of gas generator, equation (3) can be converted to the form

$$
\begin{gathered}
\frac{\partial u_{p}}{\partial z}=-\left[\left(k_{\mathrm{C}+\mathrm{O}_{2}}+2 k_{\mathrm{C}+0.5 \mathrm{O}_{2}}\right) C_{\mathrm{O}_{2}}^{w}+k_{\mathrm{C}+\mathrm{CO}_{2}} C_{\mathrm{CO}_{2}}^{w}+\right. \\
\left.+k_{\mathrm{C}+\mathrm{H}_{2} \mathrm{O}} C_{\mathrm{H}_{2} \mathrm{O}}^{w}\right] \frac{6 \mu_{\mathrm{C}}}{\rho_{p} \delta} .
\end{gathered}
$$

where $k$ - reaction rate constant; $\mu$ - molecular mass; $u_{p}, \rho_{p}$, $\delta$ - velocity, density and diameter of a particle; $C^{w}$ - surface concentration of gas components; $\tau$ - time.

The boundary condition for the ordinary differential equation (4) is $u_{p}\left(z=H_{\text {bed }}\right)=u_{p 0}$. The link between the diameter of the reacting peat particles $\delta(\tau, z)$ and their velocity $u_{p}(\tau, z)$ in the indicated zones can be obtained from the ratio of peat consumption at the outlet of the reduction zone and at the crosssection $z$ belonging to these areas [5]

$$
\frac{(1-\varepsilon) u_{p 0} \rho_{p}}{(1-\varepsilon) u_{p}(\tau, z) \rho_{p}}=\left(\frac{\delta_{0}}{\delta(\tau, z)}\right)^{3} .
$$

where $H_{\text {bed }}$ - bed height.

In such a setup of the problem, the addend should be added to the equations for thermal balance of the discrete phase [2], to account for the convective heat transfer. Based on this,

$$
\begin{gathered}
(1-\varepsilon) c_{p} \frac{\partial\left(t_{p} \rho_{p}\right)}{\partial \tau}=\frac{\partial}{\partial z}\left[\lambda_{e f f} \frac{\partial t_{p}}{\partial z}\right]+ \\
+\left[\left(k_{\mathrm{C}+\mathrm{O}_{2}} Q_{{\mathrm{C}+\mathrm{O}_{2}}_{2}}+k_{\mathrm{C}+0.5 \mathrm{O}_{2}} Q_{\mathrm{C}+0.5 \mathrm{O}_{2}}\right) C_{\mathrm{O}_{2}}^{w}-k_{\mathrm{C}+\mathrm{CO}_{2}} C_{\mathrm{CO}_{2}}^{w} Q_{\mathrm{C}+\mathrm{CO}_{2}}-\right. \\
\left.-k_{\mathrm{C}+\mathrm{H}_{2} \mathrm{O}} C_{\mathrm{H}_{2} \mathrm{O}}^{w} Q_{\mathrm{C}+\mathrm{H}_{2} \mathrm{O}}-h_{c o n v}\left(t_{p}-t_{g}\right)\right] \frac{6(1-\varepsilon)}{\delta}-Q_{p \rightarrow w}^{r a d}- \\
-Q_{p \rightarrow w}^{\text {cond }}-(1-\varepsilon) c_{p} \frac{\partial\left(t_{p} \rho_{p} u_{p}\right)}{\partial z} .
\end{gathered}
$$

where $Q$ - thermal effect of reaction, or thermal flow; $t_{p}, t_{g}-$ particle and gas temperatures; $c$ - heat capacity; $h_{\text {conv }}-$ convective heat transfer coefficient.

Converting equation (6) using (3) when $\partial \rho_{p} / \partial \tau=0$, we obtain a parabolic equation describing the change in thermal energy of the reacting peat particles within the oxidizing and reducing zones [6]

$$
\begin{gathered}
(1-\varepsilon) c_{p} \rho_{p} \frac{\partial t_{p}}{\partial \tau}=\frac{\partial}{\partial z}\left[\lambda_{\text {eff }} \frac{\partial t_{p}}{\partial z}\right]+\left[\left(k_{\mathrm{C}+\mathrm{O}_{2}} Q_{\mathrm{C}+\mathrm{O}_{2}}+\right.\right. \\
\left.+k_{\mathrm{C}+0.5 \mathrm{O}_{2}} Q_{\mathrm{C}+0.5 \mathrm{O}_{2}}\right) C_{\mathrm{O}_{2}}^{w}-k_{\mathrm{C}+\mathrm{CO}_{2}} C_{\mathrm{CO}_{2}}^{w} Q_{\mathrm{C}+\mathrm{CO}_{2}}- \\
\left.-k_{\mathrm{C}+\mathrm{H}_{2} \mathrm{O}} C_{\mathrm{H}_{2} \mathrm{O}}^{w} Q_{\mathrm{C}+\mathrm{H}_{2} \mathrm{O}}-h_{c o n v}\left(t_{p}-t_{g}\right)\right] \frac{6(1-\varepsilon)}{\delta}- \\
-Q_{p \rightarrow w}^{r a d}-Q_{p \rightarrow w}^{c o n d}-(1-\varepsilon) c_{p} \rho_{p} u_{p} \frac{\partial t_{p}}{\partial z}+ \\
+(1-\varepsilon) c_{p} t_{p}\left[\left(k_{\mathrm{C}+\mathrm{O}_{2}}+2 k_{\mathrm{C}+0.5 \mathrm{O}_{2}}\right) C_{\mathrm{O}_{2}}^{w}+k_{\mathrm{C}+\mathrm{CO}_{2}} C_{\mathrm{CO}_{2}}^{w}+\right. \\
\left.+k_{\mathrm{C}+\mathrm{H}_{2} \mathrm{O}} C_{\mathrm{H}_{2} \mathrm{O}}^{w}\right] \frac{6 \mu_{\mathrm{C}}}{\delta} .
\end{gathered}
$$

The area of dry distillation is characterized by a significant decrease in the density of the disperse phase $\rho_{p}(\tau, z)$ at $\delta(\tau, z)=$ $\left.=\delta_{0},\right) u_{p}(\tau, \mathrm{z})=u_{p 0}$ and relatively low temperatures of phases [7, 8]. In this case, the continuity equation for the disperse phase (3) can be brought to the form [9]

$$
\frac{\partial \rho_{p}}{\partial \tau}+u_{p} \frac{\partial \rho_{p}}{\partial z}=-\frac{\rho_{p 0}\left(1-V_{d}\right) V_{d} A_{\text {volat }} \exp \left[-\int_{0}^{\tau} A_{\text {volat }} d \tau\right]}{\left(1-V_{d} \exp \left[-\int_{0}^{\tau} A_{\text {volat }} d \tau\right]\right)^{2}} .
$$

where $V_{d}$ - mass fraction of light substances; $A_{\text {volat }}-$ pre-exponential factor.

The boundary conditions for the first order equation (8) [10], which belongs to the hyperbolic type, are obtained from the ratio: at the bed outlet $\rho_{p}\left(z=H_{\text {bed }}\right)=\rho_{p 0}$, and at that of the reducing zone $\rho_{p}=\rho_{p 0}\left(1-V_{d}\right)$ [11]. Further, convert a parabolic equation (6) based on (8)

$$
\begin{aligned}
& (1-\varepsilon) c_{p} \rho_{p} \frac{\partial t_{p}}{\partial \tau}+(1-\varepsilon) c_{p} u_{p} \rho_{p} \frac{\partial t_{p}}{\partial z}=\frac{\partial}{\partial z}\left[\lambda_{\text {eff }} \frac{\partial t_{p}}{\partial z}\right]+ \\
& +\left[\left(k_{\mathrm{C}+\mathrm{O}_{2}} Q_{\mathrm{C}+\mathrm{O}_{2}}+k_{\mathrm{C}+0.5 \mathrm{O}_{2}} Q_{\mathrm{C}+0.5 \mathrm{O}_{2}}\right) C_{\mathrm{O}_{2}}^{w}-k_{\mathrm{C}+\mathrm{CO}_{2}} C_{\mathrm{CO}_{2}}^{w} Q_{\mathrm{C}+\mathrm{CO}_{2}}-\right. \\
& \left.-k_{\mathrm{C}+\mathrm{H}_{2} \mathrm{O}} C_{\mathrm{H}_{2} \mathrm{O}}^{w} Q_{\mathrm{C}+\mathrm{H}_{2} \mathrm{O}}-h_{\text {conv }}\left(t_{p}-t_{g}\right)\right] \frac{6(1-\varepsilon)}{\delta}-Q_{p \rightarrow w}^{\text {rad }}- \\
& -Q_{p \rightarrow w}^{\text {cond }}+(1-\varepsilon) c_{p} t_{p} \frac{\rho_{p 0}\left(1-V_{d}\right) V_{d} A_{\text {volat }} \exp \left[-\int_{0}^{\tau} A_{\text {volat }} d \tau\right]}{\left(1-V_{d} \exp \left[-\int_{0}^{\tau} A_{v o l a t} d \tau\right]\right)^{2}} .
\end{aligned}
$$

The continuity equation for gas components can be presented in the following form.

For oxygen

$$
\begin{gathered}
\frac{\partial C_{\mathrm{O}_{2}}}{\partial \tau}+\frac{\partial\left(u_{\mathrm{g}} C_{\mathrm{O}_{2}}\right)}{\partial z}=\frac{\partial}{\partial z}\left[D_{\mathrm{O}_{2} \rightarrow \mathrm{cm}} \frac{\partial C_{\mathrm{O}_{2}}}{\partial z}\right]- \\
-\frac{6\left(k_{\mathrm{C}+\mathrm{O}_{2}}+k_{\mathrm{C}+0.5 \mathrm{O}_{2}}\right) C_{\mathrm{O}_{2}}^{\mathrm{w}}(1-\varepsilon)}{\delta}-0.5 r_{\mathrm{CO}+\mathrm{O}_{2}}-0.5 r_{\mathrm{H}_{2}+\mathrm{O}_{2}} ;
\end{gathered}
$$

carbon dioxide

$$
\begin{gathered}
\frac{\partial C_{\mathrm{CO}_{2}}}{\partial \tau}+\frac{\partial\left(u_{\mathrm{g}} C_{\mathrm{CO}_{2}}\right)}{\partial z}=\frac{\partial}{\partial z}\left[D_{\mathrm{CO}_{2} \rightarrow \mathrm{cm}} \frac{\partial C_{\mathrm{CO}_{2}}}{\partial z}\right]+ \\
+\frac{6\left(k_{\mathrm{C}+\mathrm{O}_{2}} C_{\mathrm{O}_{2}}^{w}-k_{\mathrm{C}+\mathrm{CO}_{2}} C_{\mathrm{CO}_{2}}^{w}\right)(1-\varepsilon)}{\delta}+r_{\mathrm{CO}+\mathrm{O}_{2}}+ \\
+r_{\mathrm{CO}+\mathrm{H}_{2} \mathrm{O}}+0.13 r_{\mathrm{CH}_{1.193} \mathrm{O}_{0.4083} \rightarrow} ;
\end{gathered}
$$

carbon monoxide

$$
\begin{gathered}
\frac{\partial C_{\mathrm{CO}}}{\partial \tau}+\frac{\partial\left(u_{g} C_{\mathrm{CO}}\right)}{\partial z}=\frac{\partial}{\partial z}\left[D_{\mathrm{CO} \rightarrow \mathrm{cm}} \frac{\partial C_{\mathrm{CO}}}{\partial z}\right]+ \\
+\frac{12\left[k_{\mathrm{C}+0.5 \mathrm{O}_{2}} C_{\mathrm{O}_{2}}^{w}+k_{\mathrm{C}+\mathrm{CO}_{2}} C_{\mathrm{CO}_{2}}^{w}+k_{\mathrm{C}+\mathrm{H}_{2} \mathrm{O}} C_{\mathrm{H}_{2} \mathrm{O}}^{w}\right](1-\varepsilon)}{\delta}- \\
-r_{\mathrm{CO}+\mathrm{O}_{2}}-r_{\mathrm{CO}+\mathrm{H}_{2} \mathrm{O}}+r_{\mathrm{CH}_{4}+\mathrm{H}_{2} \mathrm{O}}+0.205 r_{\mathrm{CH}_{1.193} \mathrm{O}_{0.4083} \rightarrow} ;
\end{gathered}
$$

methane

$$
\begin{gathered}
\frac{\partial C_{\mathrm{CH}_{4}}}{\partial \tau}+\frac{\partial\left(u_{g} C_{\mathrm{CH}_{4}}\right)}{\partial z}=\frac{\partial}{\partial z}\left[D_{\mathrm{CH}_{4} \rightarrow \mathrm{cm}} \frac{\partial C_{\mathrm{CH}_{4}}}{\partial z}\right]-r_{\mathrm{CH}_{4}+\mathrm{H}_{2} \mathrm{O}}+ \\
+0.33266 r_{\mathrm{CH}_{1.193} \mathrm{O}_{0.4883} \rightarrow} ;
\end{gathered}
$$

water vapor

$$
\begin{gathered}
\frac{\partial C_{\mathrm{H}_{2} \mathrm{O}}}{\partial \tau}+\frac{\partial\left(u_{g} C_{\mathrm{H}_{2} \mathrm{O}}\right)}{\partial z}=\frac{\partial}{\partial z}\left[D_{\mathrm{H}_{2} \mathrm{O} \rightarrow \mathrm{cm}} \frac{\partial C_{\mathrm{H}_{2} \mathrm{O}}}{\partial z}\right]- \\
-\frac{6 k_{\mathrm{C}+\mathrm{H}_{2} \mathrm{O}} C_{\mathrm{H}_{2} \mathrm{O}}^{w}(1-\varepsilon)}{\delta}+r_{\mathrm{H}_{2}+\mathrm{O}_{2}}-r_{\mathrm{CO}+\mathrm{H}_{2} \mathrm{O}}+0.375 r_{\mathrm{CH}_{1.193} \mathrm{O}_{0.4083} \rightarrow} ;
\end{gathered}
$$

hydrogen 


$$
\begin{gathered}
\frac{\partial C_{\mathrm{H}_{2}}}{\partial \tau}+\frac{\partial\left(u_{\mathrm{g}} C_{\mathrm{H}_{2}}\right)}{\partial z}=\frac{\partial}{\partial z}\left[D_{\mathrm{H}_{2} \rightarrow \mathrm{cm}} \frac{\partial C_{\mathrm{H}_{2}}}{\partial z}\right]+ \\
+\frac{6 k_{\mathrm{C}+\mathrm{H}_{2} \mathrm{O}} C_{\mathrm{H}_{2} \mathrm{O}}^{w}(1-\varepsilon)}{\delta}-r_{\mathrm{H}_{2}+\mathrm{O}_{2}}+r_{\mathrm{CO}+\mathrm{H}_{2} \mathrm{O}}+3 r_{\mathrm{CH}_{4}+\mathrm{H}_{2} \mathrm{O}}+ \\
+0.0256 r_{\mathrm{CH}_{1+33} \mathrm{O}_{04083} \rightarrow} ;
\end{gathered}
$$

acetylene

$$
\begin{gathered}
\frac{\partial C_{\mathrm{C}_{2} \mathrm{H}_{2}}}{\partial \tau}+\frac{\partial\left(u_{\mathrm{g}} C_{\mathrm{C}_{2} \mathrm{H}_{2}}\right)}{\partial z}=\frac{\partial}{\partial z}\left[D_{\mathrm{C}_{2} \mathrm{H}_{2} \rightarrow \mathrm{cm}} \frac{\partial C_{\mathrm{C}_{2} \mathrm{H}_{2}}}{\partial z}\right]+ \\
+0.1625 r_{\mathrm{CH}_{1.193} \mathrm{O}_{0.4083} \rightarrow} ;
\end{gathered}
$$

oxygen-containing compound $\mathrm{CH}_{1.193} \mathrm{O}_{0.4083}$

$$
\begin{gathered}
\frac{\partial C_{\mathrm{CH}_{1.193} \mathrm{O}_{0.4083}}+\frac{\partial\left(u_{g} C_{\mathrm{CH}_{1.193} \mathrm{O}_{0.4083}}\right)}{\partial \tau}=}{\partial z}= \\
=\frac{(1-\varepsilon) \rho_{p 0}\left(1-V_{d}\right) V_{d} A_{\text {volat }} \exp \left[-\int_{0}^{\tau} A_{\text {volat }} d \tau\right]}{\left(1-V_{d} \exp \left[-\int_{0}^{\tau} A_{v o l a t} d \tau\right]\right)^{2} \mu_{\mathrm{CH}_{1.193} \mathrm{O}_{0.4083}}}+ \\
+\frac{\partial}{\partial z}\left[D_{\mathrm{CH}_{1.193} \mathrm{O}_{0.4083} \rightarrow \mathrm{cm}} \frac{\left.\partial C_{\mathrm{CH}_{1.193} \mathrm{O}_{0.4083}}\right]-r_{\mathrm{CH}_{1.193} \mathrm{O}_{0.4083} \rightarrow}}{\partial z}\right]
\end{gathered}
$$

nitrogen

$$
\frac{\partial C_{\mathrm{N}_{2}}}{\partial \tau}+\frac{\partial\left(u_{g} C_{\mathrm{N}_{2}}\right)}{\partial z}=\frac{\partial}{\partial z}\left[D_{\mathrm{N}_{2} \rightarrow \mathrm{cm}} \frac{\partial C_{\mathrm{N}_{2}}}{\partial z}\right]+r_{\mathrm{N}_{2} \rightarrow} .
$$

The momentum and energy conservation equations for the gas phase are

$$
\begin{aligned}
& \frac{\partial\left(\rho_{g} u_{g}\right)}{\partial \tau}+\frac{\partial\left(\rho_{g} u_{g}^{2}\right)}{\partial z}=-\frac{\partial P_{g}}{\partial z}-g \rho_{g}+\frac{\partial}{\partial z}\left[\rho_{g} \eta_{g} \frac{\partial u_{g}}{\partial z}\right]- \\
& -\frac{150 \eta_{g} \rho_{g}(1-\varepsilon)^{2} u_{g}}{\delta^{2} \varepsilon^{3}}-\frac{1.75 \rho_{g}(1-\varepsilon) u_{g}^{2}}{\delta \varepsilon^{3}} \\
& \frac{\partial\left(\rho_{g} c_{g} t_{g}\right)}{\partial \tau}+\frac{\partial\left(\rho_{g} u_{g} c_{g} t_{g}\right)}{\partial z}=\frac{\partial}{\partial z}\left[\lambda_{g, e f f} \frac{\partial t_{g}}{\partial z}\right]+ \\
& +h_{\text {conv }}\left(t_{p}-t_{g}\right) \frac{6(1-\varepsilon)}{\delta}+r_{\mathrm{CO}+\mathrm{O}_{2}} Q_{\mathrm{CO}+\mathrm{O}_{2}}+r_{\mathrm{H}_{2}+\mathrm{O}_{2}} Q_{\mathrm{H}_{2}+\mathrm{O}_{2}}+ \\
& +r_{\mathrm{CO}+\mathrm{H}_{2} \mathrm{O}} Q_{\mathrm{CO}+\mathrm{H}_{2} \mathrm{O}}+r_{\mathrm{CH}_{4}+\mathrm{H}_{2} \mathrm{O}} Q_{\mathrm{CH}_{4}+\mathrm{H}_{2} \mathrm{O}},
\end{aligned}
$$

where $D_{j} \rightarrow \mathrm{cm}-$ diffusion coefficient of the $j$ gas component into the mixture; $u_{g}, \eta_{g}$ - velocity and kinematic gas viscosity; $g$ - acceleration of gravity; $\lambda_{\text {eff }}$ is the effective coefficient of thermal conductivity, $C_{\mathrm{O}_{2}}^{w}, C_{\mathrm{CO}_{2}}^{w}, C_{\mathrm{H}_{2} \mathrm{O}}^{w}$ and $C_{\mathrm{CO}}^{w}$, the concentration of gas components on the surface of the particle,

$r_{\mathrm{H}_{2}+\mathrm{O}_{2}}, r_{\mathrm{CO}+\mathrm{H}_{2} \mathrm{O}}, r_{\mathrm{CO}+\mathrm{O}_{2}}, r_{\mathrm{CH}_{4}+\mathrm{H}_{2} \mathrm{O}}, r_{\mathrm{N}_{2} \rightarrow}$ and $r_{\mathrm{CH}_{1.193} \mathrm{O}_{0.4083} \rightarrow}$ are the reaction rates; along with the yield of volatile matter from peat, they are determined in accordance with [12].

Boundary conditions for parabolic equations (7), (9-20) at the bed inlet $(z=0)$ are determined from

$$
\begin{gathered}
\frac{\partial t_{\mathrm{p}}}{\partial z}=C_{\mathrm{CO}_{2}}=C_{\mathrm{CO}}=C_{\mathrm{CH}_{4}}=C_{\mathrm{H}_{2}}=C_{\mathrm{C}_{2} \mathrm{H}_{2}}= \\
=C_{\mathrm{CH}_{1.193} \mathrm{O}_{0.4083}}=0 ; \quad C_{\mathrm{O}_{2}}=C_{\mathrm{O}_{2} 0} ; \\
C_{\mathrm{H}_{2} \mathrm{O}}=C_{\mathrm{H}_{2} \mathrm{O} 0} ; \quad C_{\mathrm{N}_{2}}=C_{\mathrm{N}_{2} 0} ; \quad u_{g}=u_{g 0} ; \quad t_{g}=t_{g 0},
\end{gathered}
$$

at the outlet from the bed $\left(z=H_{b e d}\right)$

$$
\begin{gathered}
\frac{\partial t_{g}}{\partial z}=\frac{\partial C_{\mathrm{O}_{2}}}{\partial z}=\frac{\partial C_{\mathrm{N}_{2}}}{\partial z}=\frac{\partial C_{\mathrm{CO}_{2}}}{\partial z}=\frac{\partial C_{\mathrm{CO}}}{\partial z}=\frac{\partial C_{\mathrm{CH}_{4}}}{\partial z}=\frac{\partial C_{\mathrm{H}_{2} \mathrm{O}}}{\partial z}= \\
=\frac{\partial C_{\mathrm{H}_{2}}}{\partial z}=\frac{\partial C_{\mathrm{C}_{2} \mathrm{H}_{2}}}{\partial z}=\frac{\partial C_{\mathrm{CH}_{1.193} \mathrm{O}_{0.4083}}}{\partial z}=\frac{\partial u_{g}}{\partial z}=0 ;
\end{gathered}
$$

$$
t_{p}=t_{p}\left(z=H_{b e d}\right) .
$$

The first and second order equations are integrated applying the marching method of direct and reverse runs on an uneven grid, which is thickened in the lower part of the bed [13, 14]. In this case, for the solution of parabolic equations (7), (9-20), the method of iterations was used, while there was no need to use iterations for hyperbolic equations. The pressure gradient was excluded with the well-known SIMPLE method [15].

Mathematical description of motion, heat transfer and combustion of gas coal dust particles and synthetic peat gas in the combustion chamber of TPP-210A boiler. Modeling of the combustion process of fine coal dust and generator gas is carried out using ANSYS FLUENT (Non-Premixed Combustion model). This model applies the Euler-Lagrange description of the medium movement: the mass, momentum and heat transfer of the gas phase is presented in Euler variables, and the equation of change in mass, motion and energy of the discrete phase - in Lagrange variables. To complete the carrier medium transmission equations, a two-parameter $k-\varepsilon$ model of turbulence is used, and description of the parameters of disperse phase along the trajectories of individual particles is based on Langevin type equations. Radiation heat exchange in a twophase flow is presented within the framework of the $\mathrm{P} 1 \mathrm{mo-}$ del - approximation of the method of spherical harmonics.

The system of averaged equations of transfer of the gas phase in Euler representation within the framework of intermediate continuums can be written in the form:

continuity equation for the gas mixture

$$
\frac{\partial\left(u_{g i} \rho_{g}\right)}{\partial x_{j}}=S_{m}
$$

for the component of the gas mixture $C_{k}$

$$
\frac{\partial\left(u_{\mathrm{g} j} C_{k}\right)}{\partial x_{j}}=\frac{\partial}{\partial x_{j}}\left(D \frac{\partial C_{k}}{\partial x_{j}}-<C_{k}^{\prime} u_{\mathrm{g} j}^{\prime}>\right)+S_{\mathrm{m} k}+r_{k}
$$

motion equation

$$
\frac{\partial\left(u_{g j} u_{g i} \rho_{g}\right)}{\partial x_{j}}=-\frac{\partial P}{\partial x_{i}}+\frac{\partial \tau_{i j}}{\partial x_{j}}+\frac{\partial\left(-\rho_{g}<u_{g j}^{\prime} u_{g i}^{\prime}>\right)}{\partial x_{j}}+F_{i}+S_{m o m} ;
$$

energy equation

$$
\frac{\partial\left(c_{g} \rho_{g} t_{g} u_{g j}\right)}{\partial x_{j}}=\frac{\partial}{\partial x_{j}}\left[c_{g} \rho_{g}\left(\frac{\eta_{g}}{\operatorname{Pr}_{g}} \frac{\partial t_{g}}{\partial x_{j}}-<t_{g}^{\prime} u_{g j}^{\prime}>\right)\right]+S_{r a d}+S_{h} ;
$$

pulsation energy $k_{g}$

$$
\begin{gathered}
\frac{\partial\left(\rho_{g} u_{g j} k_{g}\right)}{\partial x_{j}}=\frac{\partial}{\partial x_{i}}\left[\rho_{g}\left(\frac{\eta_{t, g}}{\sigma_{k}}+\eta_{g}\right) \frac{\partial k_{g}}{\partial x_{i}}\right]+ \\
+\frac{\rho_{g} \eta_{t, g}}{2}\left(\frac{\partial u_{g i}}{\partial x_{j}}+\frac{\partial u_{g j}}{\partial x_{i}}\right)^{2}-\rho_{g} \varepsilon_{g, d i s}-\frac{g_{j} \eta_{t, g}}{\operatorname{Pr}_{t, g}} \frac{\partial \rho_{g}}{\partial x_{j}} ;
\end{gathered}
$$

dissipative function $\varepsilon_{g, \text { dis }}$

$$
\begin{gathered}
\frac{\partial\left(\rho_{g} u_{g j} \varepsilon_{g, d i s}\right)}{\partial x_{j}}=\frac{\partial}{\partial x_{i}}\left[\rho_{g}\left(\frac{\eta_{t, g}}{\sigma_{\varepsilon}}+\eta_{g}\right) \frac{\partial \varepsilon_{g, d i s}}{\partial x_{i}}\right]+ \\
+A_{1 \varepsilon} \frac{\rho_{g} \eta_{t, g} \varepsilon_{g, d i s}}{2 k_{g}}\left(\frac{\partial u_{g i}}{\partial x_{j}}+\frac{\partial u_{g j}}{\partial x_{i}}\right)^{2}- \\
-A_{1 \varepsilon} A_{3 \varepsilon} \frac{g_{j} \eta_{t, g} \varepsilon_{g, d i s}}{\operatorname{Pr}_{t, g} k_{g}} \frac{\partial \rho_{g}}{\partial x_{i}}-A_{2 \varepsilon} \rho_{g} \frac{\varepsilon_{g, d i s}^{2}}{k_{g}} .
\end{gathered}
$$

where $S_{m}, S_{m j}, S_{m o m}$ - transition from solid phase to gas as a result of heterogeneous reactions; $S_{h}$ - change of gas enthalpy during transition of solid phase to gas phase in heterogeneous reactions; $S_{\text {rad }}-$ radiant heat transfer; $A_{1 \varepsilon}, A_{2 \varepsilon}, A_{3 \varepsilon}, \sigma_{k}, \sigma_{\varepsilon}-$ empirical constants; $F$ - force, N. 
Second moments $\left\langle C_{k}^{\prime} u_{\mathrm{g} j}^{\prime}\right\rangle,\left\langle t_{\mathrm{g}}^{\prime} u_{\mathrm{g}}^{\prime}\right\rangle,\left\langle u_{\mathrm{g} j}^{\prime} u_{\mathrm{g} i}^{\prime}\right\rangle$, appearing in equations (24-26) are calculated on the basis of the Boussinesq hypothesis

$$
\begin{gathered}
\left\langle C_{k}^{\prime} u_{g j}^{\prime}\right\rangle=-D_{t, g} \frac{\partial C_{k}}{\partial x_{j}} ;\left\langle t_{g}^{\prime} u_{g j}^{\prime}\right\rangle=-\frac{\eta_{t, g}}{\operatorname{Pr}_{t, g}} \frac{\partial t_{g}}{\partial x_{j}} ; \\
\left\langle u_{g j}^{\prime} u_{g i}^{\prime}\right\rangle=-\eta_{t, g}\left(\frac{\partial u_{g i}}{\partial x_{j}}+\frac{\partial u_{g j}}{\partial x_{i}}\right)+\frac{2}{3} k_{g} \delta_{i j} .
\end{gathered}
$$

The equation of the change in mass, motion and energy of the solid phase along the trajectories of individual particles in the Lagrange representation are written as:

motion equation

$$
\begin{gathered}
m_{p} \frac{\partial \vec{V}_{p}}{\partial \tau}=0.5 C_{D} \rho_{g} \frac{\pi \delta_{p}^{2}}{4}\left|\vec{V}_{g}-\vec{V}_{p}\right|\left(\vec{V}_{g}-\vec{V}_{p}\right)+ \\
+0.125 \pi \rho_{g} \delta_{p}^{3}\left[\left(\vec{V}_{g}-\vec{V}_{p}\right) \times \vec{\omega}_{p}\right]+\vec{F}_{S a f}+\vec{F}_{T F}-\frac{\delta_{p}^{2} P l}{t_{g}} \nabla t_{g} ;
\end{gathered}
$$

balance of mass

$$
\begin{aligned}
\frac{\partial m_{p}}{\partial \tau} & =\frac{\partial m_{\mathrm{C}+\mathrm{O}_{2}}}{\partial \tau}+\frac{\partial m_{\mathrm{C}+0.5 \mathrm{O}_{2}}}{\partial \tau}+\frac{\partial m_{\mathrm{C}+\mathrm{CO}_{2}}}{\partial \tau}+ \\
& +\frac{\partial m_{\mathrm{C}+\mathrm{H}_{2} \mathrm{O}}}{\partial \tau}+\frac{\partial m_{\text {volat }}}{\partial \tau}+\frac{\partial m_{\text {vapor }}}{\partial \tau},
\end{aligned}
$$

where the first four terms in the left hand side of equation (31) characterize the transition of the solid phase into the gas phase through the heterogeneous reactions $\mathrm{C}+\mathrm{O}_{2}=\mathrm{CO}_{2}$, $\mathrm{C}+0.5 \mathrm{O}_{2}=\mathrm{CO}, \mathrm{C}+\mathrm{CO}_{2}=2 \mathrm{CO}$ and $\mathrm{C}+\mathrm{H}_{2} \mathrm{O}=\mathrm{CO}+\mathrm{H}_{2}$. These components are determined similar to (3). The fifth and sixth terms of the equation are the weight loss rate due to the yield of pyrolysis gases [16] and the evaporation of moisture [17].

Equation of particles energy

$$
\begin{gathered}
c_{p} m_{p} \frac{\partial t_{p}}{\partial \tau}=h_{\text {conv }}\left(t_{g}-t_{p}\right) \pi \delta_{p}^{2}+a_{p} \pi \delta_{p}^{2} \sigma_{0}\left(\theta_{R}^{4}-T_{p}^{4}\right)+Q_{\Sigma} ; \\
\theta_{R}=\left(G / 4 \sigma_{0}\right)^{1 / 4} ; \quad G=\int_{0}^{4 \pi} I d \Omega ; \\
Q_{\Sigma}=\frac{\partial m_{\mathrm{C}+\mathrm{O}_{2}}}{\partial \tau} Q_{\mathrm{C}+\mathrm{O}_{2}}+\frac{\partial m_{\mathrm{C}+0.5 \mathrm{O}_{2}}}{\partial \tau} Q_{\mathrm{C}+0.5 \mathrm{O}_{2}}+ \\
+\frac{\partial m_{\mathrm{C}+\mathrm{CO}_{2}}}{\partial \tau} Q_{\mathrm{C}+\mathrm{CO}_{2}}+\frac{\partial m_{\mathrm{C}+\mathrm{H}_{2} \mathrm{O}}}{\partial \tau} Q_{\mathrm{C}+\mathrm{H}_{2} \mathrm{O}} .
\end{gathered}
$$

$\omega-$ angular velocity; $\Omega$ - directing cosine of radiation; $\delta_{i j}-$ Kronecker symbol; $\sigma_{0}-$ Stefan-Boltzmann constant; $\tau_{i j}-$ Reynolds stress tensor component; $C_{D}-$ aerodynamic drag coefficient; $m$ - mass, кг; $a$ - emissivity factor; ); $I-$ emission intensity of gases; $l$ - the length of the mixing path.

Results. We will discuss four options of calculating gasification of particles of Volyn peat with a diameter $\delta_{0}=0.009 \mathrm{~m}$ in a steam-air mixture enriched with oxygen, in a stationary bed with the content of fixed carbon (per working mass) $\mathrm{C}_{f}^{r}=18.5124 \%$, volatile matter $\mathrm{C}_{\text {volat }}^{r}=17.4876 \%$, sulfur $\mathrm{S}^{r}=0.69 \%$, hydrogen $\mathrm{H}^{r}=3.58 \%$, oxygen $\mathrm{O}^{r}=19.6 \%$, nitrogen $\mathrm{N}^{r}=1.36 \%$, moisture $\mathrm{W}^{\mathrm{r}}=18.9 \%$, ash $\mathrm{A}^{r}=19.9 \%$, volatile substances (per dry ash mass) $V^{\text {daf }}=69.8 \%$, temperature of ash deformation and softening $t_{A \text {, def }}=1360{ }^{\circ} \mathrm{C}$ and $t_{A \text {, soft }}>$ $>1400{ }^{\circ} \mathrm{C}$, with particles density $\rho_{p 0}=1450 \mathrm{~kg} / \mathrm{m}^{3}$, porosity $\varepsilon=0.4$ under pressure $P_{g}=1.5 \mathrm{MPa}$. Option 1 (per single gasifier): steam consumption $B_{\mathrm{H}_{2} \mathrm{O}}=667 \mathrm{~kg} / \mathrm{h}$, oxygen $B_{\mathrm{O}_{2}}=$ $=1795 \mathrm{~kg} / \mathrm{h}$, nitrogen $B_{\mathrm{N}_{2}}=2667 \mathrm{~kg} / \mathrm{h}, u_{p 0}=-0.000365 \mathrm{~m} / \mathrm{s}$, $u_{p 0}=0.039 \mathrm{~m} / \mathrm{s}$, layer diameter $D_{\text {bed }}=3.446 \mathrm{~m}, B_{p}=9646 \mathrm{~kg} / \mathrm{h}$, $H_{\text {bed }}=1.1064 \mathrm{~m}$; Option 2 (per gasifier): $B_{\mathrm{H}_{2} \mathrm{O}}=667 \mathrm{~kg} / \mathrm{h}$ $B_{\mathrm{O}_{2}}=1795 \mathrm{~kg} / \mathrm{h}, \quad B_{\mathrm{N}_{2}}=2667 \mathrm{~kg} / \mathrm{h}, u_{p 0}=-0.000354 \mathrm{~m} / \mathrm{s}, u_{g 0}$
$=0.039 \mathrm{~m} / \mathrm{s}$, bed diameter $D_{\text {bed }}=3.446 \mathrm{~m}, H_{\text {bed }}=1.1064 \mathrm{~m}$; Option 3 (per gasifier): $B_{\mathrm{H}_{2} \mathrm{O}}=667 \mathrm{~kg} / \mathrm{h}, B_{\mathrm{O}_{2}}=1795 \mathrm{~kg} / \mathrm{h}$, $B_{\mathrm{N}_{2}}=2667 \mathrm{~kg} / \mathrm{h}, u_{\mathrm{p} 0}=-0.0003 \mathrm{~m} / \mathrm{s}, u_{\mathrm{g} 0}==0.039 \mathrm{~m} / \mathrm{s}, D_{\text {bed }}=$ $=3.446 \mathrm{~m}, B_{p}==9646 \mathrm{~kg} / \mathrm{h}, H_{\text {bed }}=1.1064 \mathrm{~m}$; Option 4 (per gasifier): $B_{\mathrm{H}_{2} \mathrm{O}}=1230 \mathrm{~kg} / \mathrm{h}, B_{\mathrm{O}_{2}}=1659 \mathrm{~kg} / \mathrm{h}, B_{\mathrm{N}_{2}}=2459 \mathrm{~kg} / \mathrm{h}$, $u_{p 0}=-0.000365 \mathrm{~m} / \mathrm{s}, u_{\mathrm{g} 0}=0.042 \mathrm{~m} / \mathrm{s}, D_{b e d}=3.446 \mathrm{~m}, B_{p}=$ $=9646 \mathrm{~kg} / \mathrm{h}, H_{\mathrm{bed}}=1.1064 \mathrm{~m}$. Temperature of particles at the outlet of the reactor $t_{p}\left(z=H_{\mathrm{bed}}\right)=300{ }^{\circ} \mathrm{C}$.

In Fig. 2, temperature profiles of phases are illustrated for Option 1, for the inlet fuel particles velocity $u_{p 0}=$ $=-0.000365 \mathrm{~m} / \mathrm{s}$. It is seen that the maximum heat generation zone is adjacent to a grate. In this zone, the temperature of gas and that of particles are steeply increasing and reach the maximum values of $t_{p, \max }=1114-1126$ and $t_{g, \max }=799.5-866{ }^{\circ} \mathrm{C}$ (Fig. 2). In the range of temperature under discussion, $t_{\mathrm{p}, \max }$ is lower than the temperature of the deformation of ash $t_{A}=$ $=1360{ }^{\circ} \mathrm{C}$, which allows organizing the process of peat gasification with wet slag removal in the reactor.

The time interval $0<\tau<2200 \mathrm{~s}$ is characterized by nonstationary (unsteady) process of thermochemical processing of Volyn peat with distributions of phase temperature and concentration of gas components varying in time. In the oxygen zone $0<z<0.00146 \mathrm{~m}$, there is a sharp increase in the $t_{p}(\tau, z)$ dependence due to prevailing of heat dissipation in oxidative heterogeneous reactions $\mathrm{C}+\mathrm{O}_{2}=\mathrm{CO}_{2}$ and $\mathrm{C}+0.5 \mathrm{O}_{2}=\mathrm{CO}$ and afterburning of gasification products $\mathrm{CO}$ and $\mathrm{H}_{2}(\mathrm{CO}+$ $+0.5 \mathrm{O}_{2}=\mathrm{CO}_{2}$ and $\mathrm{H}_{2}+0.5 \mathrm{O}_{2}=\mathrm{H}_{2} \mathrm{O}$ ) over the heat absorption
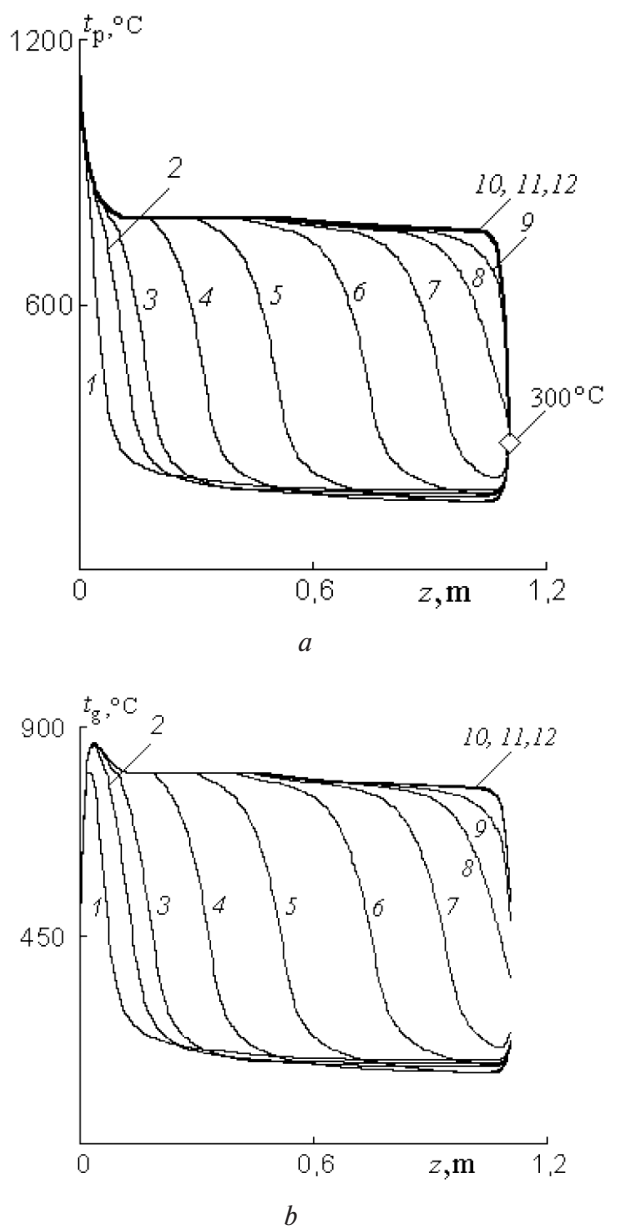

Fig. 2. Distribution of Volyn peat particle temperatures (a) and gas (b) through the height of a stationary bed versus time for Option 1:

$1-100 s ; 2-200 s ; 3-300 s ; 4-600 s ; 5-1000 s ; 6-1500 s$ $7-1900 s ; 8-2200 s ; 9-2400 s ; 10-2800 s ; 11-3000 s$ $12-3200 s$ 
in reducing reactions $\mathrm{C}+\mathrm{CO}_{2}=2 \mathrm{CO}$ and $\mathrm{C}+\mathrm{H}_{2} \mathrm{O}=\mathrm{CO}+\mathrm{H}_{2}$, resulting in that the temperature of the reacting particles at the outlet of the zone reaches maximum value of $t_{p, \max }=1126{ }^{\circ} \mathrm{C}$ (curve 3 in Fig. 2).

The contents of $\mathrm{O}_{2}$ and $\mathrm{H}_{2} \mathrm{O}$ in gas drop from 30 and $19.6 \%$ to 24.9 and $16.9 \%$, respectively, the volumetric fraction of gas components $\mathrm{CO}_{2}, \mathrm{CO}$ and $\mathrm{H}_{2}$ increase and achieve the values: $R_{\mathrm{CO}_{2}}=0.106, R_{\mathrm{CO}}=8.33$ and $R_{\mathrm{H}_{2}}=1.78 \%$ (curves 3 in Figs. 3, $a, b, 4, a)$.

In the oxidation-reducing region, $0.00146<z<0.0361 \mathrm{~m}$, oxygen content $R_{\mathrm{O}_{2}} \rightarrow 1 \%$, the heat absorption in reducing heterogeneous reactions prevails over the release of heat in oxidative reactions. Values $t_{p}$ and $R_{\mathrm{H}_{2} \mathrm{O}}$ decrease to $878{ }^{\circ} \mathrm{C}$ and $1.54 \%$, the volumetric fractions of gas components increase and reach the following values $R_{\mathrm{CO}_{2}}=2.1, R_{\mathrm{CO}}=47.704$ and $R_{\mathrm{H}_{2}}=12.26 \%$.

Gasification zone, $0.0361<z<0.171 \mathrm{~m}$, is characterized by the absence of pyrolysis gases, reduction of the volume ratios of water vapor, carbon dioxide and of the temperature of peat particles to the levels of $R_{\mathrm{H}_{2} \mathrm{O}}=0.043 \%, R_{\mathrm{CO}_{2}}=1.86 \%$, and

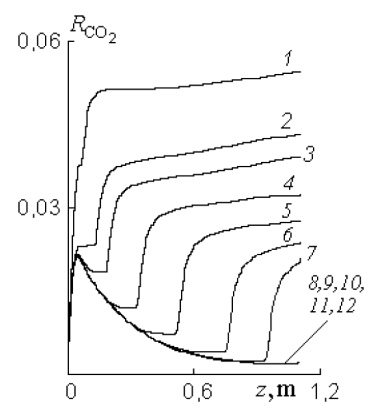

$a$

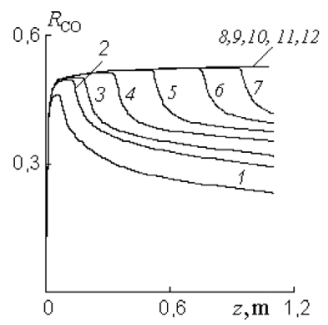

b

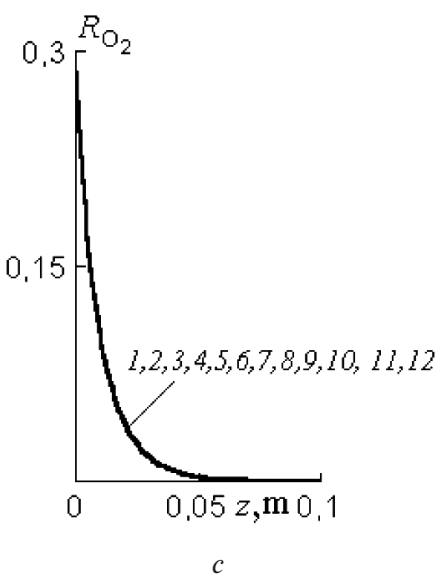

Fig. 3. Distribution of volumetric fractions of gas components $\mathrm{CO}_{2}(a), \mathrm{CO}(b)$ and $\mathrm{O}_{2}(c)$ through the height of a stationary bed versus time for Option 1:

$1-100 s ; 2-200 s ; 3-300 s ; 4-600 s ; 5-1000 s ; 6-1500 s$; $7-1900 s ; 8-2200 s ; 9-2400 s ; 10-2800 s ; 11-3000 s ;$ $12-3200 s$ $t_{p}=484.5^{\circ} \mathrm{C}$, the weak growth of values $R_{\mathrm{CO}}=50.23$ and $R_{\mathrm{H}_{2}}=13.4 \%$. Thus it follows that the most extended gasification zone with a small degree of carbon conversion deems inefficient.

In the dry distillation zone, $0.171<z<1.1064 \mathrm{~m}$, heating of peat particles to a temperature of $168-484.5^{\circ} \mathrm{C}$ (curve 3 in Fig. 2) occurs due to interphase convective heat transfer and radiation-conductive heat transfer of the disperse phase. For this reason, there is an intense yield of volatile substances in the form of an oxygen-containing compound $\mathrm{CH}_{1.193} \mathrm{O}_{0.4083} \rightarrow$ $\rightarrow 0.205 \mathrm{CO}+0.13 \mathrm{CO}_{2}+0.33266 \mathrm{CH}_{4}+0.1625 \mathrm{C}_{2} \mathrm{H}_{2}+$ $+0.0256 \mathrm{H}_{2}+0.375 \mathrm{H}_{2} \mathrm{O}$ with its subsequent partial decomposition to gas components $\mathrm{CO}, \mathrm{CO}_{2}, \mathrm{CH}_{4}, \mathrm{C}_{2} \mathrm{H}_{2}, \mathrm{H}_{2}$ and $\mathrm{H}_{2} \mathrm{O}$, which provides growth of the functions $R_{\mathrm{CH}_{4}}(\tau, z)$, $R_{\mathrm{C}_{2} \mathrm{H}_{2}}(\tau, z), \quad R_{\mathrm{CH}_{1.193} \mathrm{O}_{0.4083}}(\tau, z), \quad R_{\mathrm{H}_{2} \mathrm{O}}(\tau, z)$ and $R_{\mathrm{CO}_{2}}(\tau, z)$ and downslope in dependencies $R_{\mathrm{CO}}(\tau, z)$ and $R_{\mathrm{H}_{2}}(\tau, z)$ in the pyrolysis zone (curves 3 in Figs. 3, $a, b, 4, a, b$ and 5). Such behavior of the curves can be explained by the following reasons. In the dry distillation zone, there is a mixing of two gas streams: of pyrolysis gas $\left(\mathrm{CO}, \mathrm{CO}_{2}, \mathrm{CH}_{4}, \mathrm{C}_{2} \mathrm{H}_{2}, \mathrm{H}_{2}, \mathrm{H}_{2} \mathrm{O}\right.$ and $\mathrm{CH}_{1.193} \mathrm{O}_{0.4083}$ ), in which the amount of $\mathrm{CH}_{4}$ and $\mathrm{H}_{2} \mathrm{O}$ prevails over $\mathrm{CO}$ and $\mathrm{H}_{2}$ (First Reaction (2)), and of the synthesis gas coming from the reduction zone, which predominantly consists of $\mathrm{CO}, \mathrm{N}_{2}, \mathrm{H}_{2}$ and a small volumetric fraction of $R_{\mathrm{CO}}$.

Therefore, upon mixing of two gas flows the decrease occurs of values $R_{\mathrm{CO}}$ and $R_{\mathrm{H}_{2}}$, while the values $R_{\mathrm{CH}_{4}}, R_{\mathrm{CO}_{2}}$, $R_{\mathrm{H}_{2} \mathrm{O}}$ and $R_{\mathrm{C}_{2} \mathrm{H}_{2}}$ are increasing.

Intense yield of volatile matter contributes to a decrease in particle density with unchanged diameter and velocity of the disperse phase (curves 3 in Figs. 4 and 6). In the time interval $0<\tau<2200 \mathrm{~s}$ in the area of $0.192<z<1.1064 \mathrm{~m}$ the function $\rho_{p}(\tau, z)$ decreases dramatically due to the yield of volatile matter from a value of 1050 to $556.6 \mathrm{~kg} / \mathrm{m}^{3}$, while in the range $0.0361<z<0.171 \mathrm{~m}$ the particle density does not change $\rho_{p}(\tau, z)=556.6 \mathrm{~kg} / \mathrm{m}^{3}$ (curve 3 in Fig. 6). In the range of $2200 \leq \tau<3200 \mathrm{~s}$, where a dry distillation area is absent, the density is $\rho_{p}(\tau, z)=556.6 \mathrm{~kg} / \mathrm{m}^{3}$ (curves $8-12$ ).

In Fig. 6 , the distribution of peat particle velocities is presented through the height of the fixed bed versus time for Option 1. From the comparison of Figs. 4 and 6 it follows that the disperse-phase velocity profiles $u_{p}(\tau, z)$ resemble the mirror reflection of curves $\delta(\tau, z)$. Indeed, in the pyrolysis zone, the values of functions remain unchanged: $u_{p}(\tau, z)=u_{p 0}$ and $\delta(\tau$, $z)=\delta_{0}$.

In oxygen, oxidation-reducing zones and in the gasification zone, where an intensive conversion of ash-coke particles occurs with a change in their diameter and speed through heterogeneous oxidation and reduction reactions, the values $\delta_{0}=$ $=0.009 \mathrm{~m}$ and $u_{p 0}=-0.000365 \mathrm{~m} / \mathrm{s}$ decrease to $\delta_{e}=7.24 \mathrm{~mm}$ and $u_{p e}=-0.00019 \mathrm{~m} / \mathrm{s}$.

In the time interval $2200 \leq \tau<3200 \mathrm{~s}$ profiles of each function $t_{p}(\tau, z), t_{g}(\tau, z), R_{\mathrm{CO}}(\tau, z), R_{\mathrm{CO}_{2}}(\tau, z), \quad R_{\mathrm{H}_{2}}(\tau, z)$, $R_{\mathrm{H}_{2} \mathrm{O}}(\tau, z), \rho_{p}(\tau, z), \delta(\tau, z)$ and $q_{\text {goruch }}(\tau, z)$ merge into a single curve, indicating the setting of a stationary state of the process (curves 8-12 in Figs. 2, $3 a, b, 4,6, a, b)$. With such an organization of the working process, the spare temperature range of the grate slagging is quite large $t_{A}-t_{p, \max }=234-$ $246{ }^{\circ} \mathrm{C}$, and the diameter of ash-coke particles at the outlet of the bed is $\delta_{e}=7.24 \mathrm{~mm}$ (Fig. 4, c), which is slightly higher than the diameter of the ash particles $\delta_{a s h}=7.23 \mathrm{~mm}$, indicating a low mechanical unburnt matter $q_{4,1}=0.13-0.3 \%$ (Fig. 6,c).

At the outlet of the reactor, the generator gas with the following parameters is obtained for Option 1 (per dry mass): $R_{\mathrm{CO}}=33.474 \%, \quad R_{\mathrm{H}_{2}}=8.26 \%, \quad R_{\mathrm{H}_{2} \mathrm{O}}=7.82 \%, \quad R_{\mathrm{CO}_{2}}=$ $=3.672 \%, R_{\mathrm{CH}_{4}}=6.94 \%, R_{\mathrm{N}_{2}}=19.81 \%, R_{\mathrm{CH}_{1.193} \mathrm{O}_{0.4083}}(\tau, z)=$ $=16.63 \%$ and $R_{\mathrm{C}_{2} \mathrm{H}_{2}}=3.39 \%$ at $\tau=400 \mathrm{~s}$; and $R_{\mathrm{CO}}=52.8 \%$, $R_{\mathrm{H}_{2}}=13.21 \%, R_{\mathrm{N}_{2}}=33.88 \%$ at $\tau=3200 \mathrm{~s}$. 

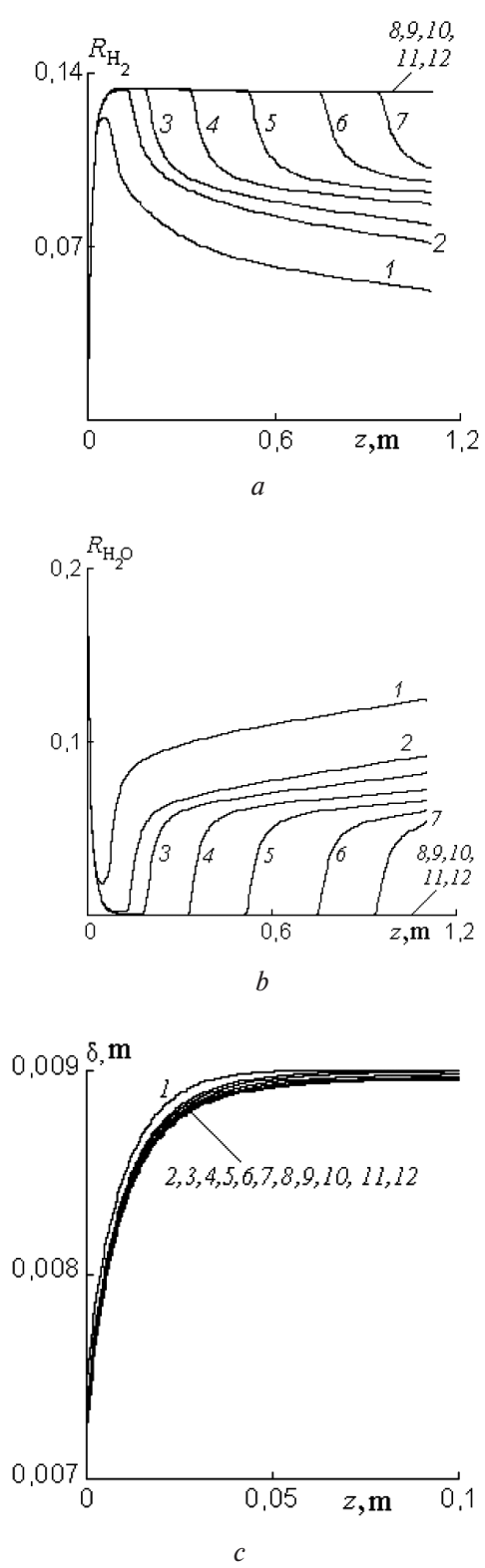

Fig. 4. Distribution of volumetric fractions of gas components $\mathrm{H}_{2}$ (a) and $\mathrm{H}_{2} \mathrm{O}(b)$, particle diameter (c) through the height of a stationary bed versus time for Option 1:

$1-100 s ; 2-200 s ; 3-300 s ; 4-600 s ; 5-1000 s ; 6-1500 s$; $7-1900 s ; 8-2200 s ; 9-2400 s ; 10-2800 s ; 11-3000 s$; $12-3200 s$

The raw synthetic gas in the amount of $33005 \mathrm{~kg} / \mathrm{h}$, obtained in stationary mode ( $\tau \geq 2200 \mathrm{~s})$ of thermochemical processing of $28939 \mathrm{~kg} / \mathrm{h}$ of raw peat in three reactors, is directed to chamber 17 (Fig. 1), where the condensation of heavy hydrocarbons $\mathrm{C}_{2} \mathrm{H}_{2}$ in the quantity $\sum_{i=1}^{3} B_{\mathrm{C}_{2} \mathrm{H}_{2} i}=1754.5 \mathrm{~kg} / \mathrm{h}$ and cleaning from disperse particles occurs. Cleared from tar and dust, the peat gas is mixed with $5469.5 \mathrm{~kg} / \mathrm{h}$ of evaporated moisture of the raw peat 26 and after reset of pressure in the gas turbine 34 enters gas burners 8 (Fig. 1). The mass fractions of the gas components of peat gas at the inlet to the furnace can be presented as: $\mathrm{H}_{2} \mathrm{O}=0.226, \mathrm{H}_{2}=0.00723, \mathrm{CO}=0.405$, $\mathrm{CO}_{2}=0.0674, \mathrm{CH}_{4}=0.0608, \mathrm{H}_{2} \mathrm{~S}=0.00472, \mathrm{~N}_{2}=0.229$.

In the furnace of TPP-210A boiler $7,36720 \mathrm{~kg} / \mathrm{h}$ of synthetic gas (20\% by fuel energy) and $48996 \mathrm{~kg} / \mathrm{h}$ of fine coal dust are supplied to the main burners 5 (Fig. 1) for joint combustion. This process in greater detail shall be examined in the third part of this work.

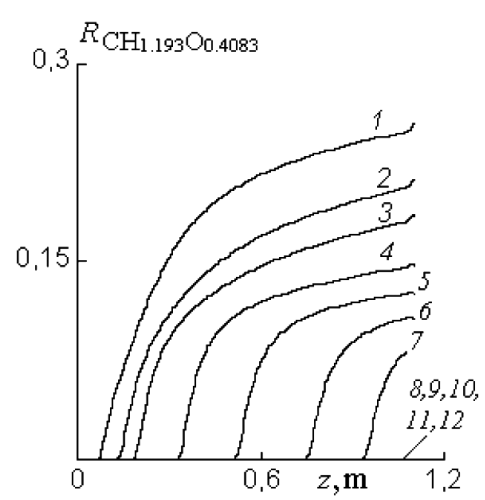

$a$

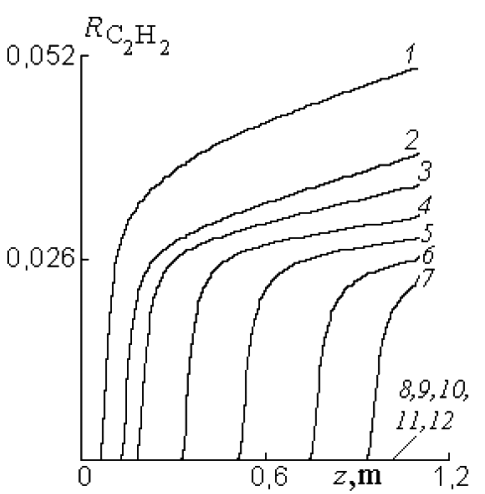

$b$

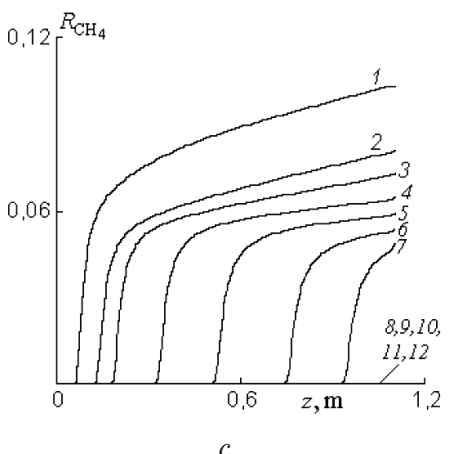

Fig. 5. Distribution of volumetric particles of gas components of oxygen-containing compound (a), acetylene (b) and methane (c) through the height of a stationary bed versus time for Option 1:

$1-100 s ; 2-200 s ; 3-300 s ; 4-600 s ; 5-1000 s ; 6-1500 s$; $7-1900 s ; 8-2200 s ; 9-2400 s ; 10-2800 s ; 11-3000 s$; $12-3200 s$

Conclusions. A two-dimensional system of parabolic and hyperbolic equations is constructed describing aerodynamics, thermal and mass exchange and chemical reaction of the multiphase medium in a fixed bed. The main difference from the existing models is the accounting of the velocity of particles, its variation in the oxidative and reducing zones due to a decrease in the diameter of the disperse phase in the course of the heterogeneous reactions.

The modeling of the combustion process of gas-disperse multiphase mixture is performed using ANSYS FLUENT (NonPremixed Combustion model). This model incorporates the Euler-Lagrange description of the medium movement: the transfer of mass, momentum and heat of the gas phase is presented in Euler variables, and the equation of the change in mass, motion and energy of the discrete phase - in Lagrange variables.

The developed model allows obtaining detailed information on the structural characteristics of the gas generator in a fixed 


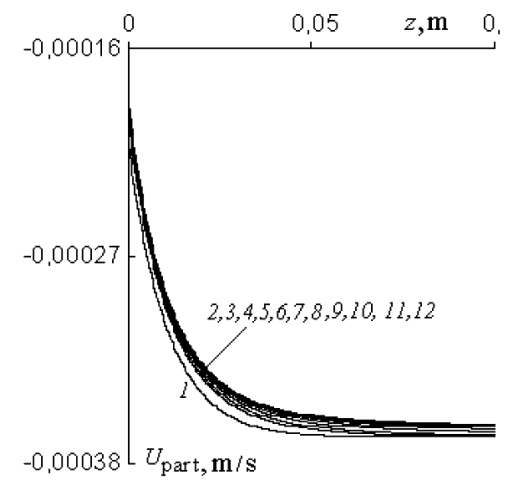

$a$
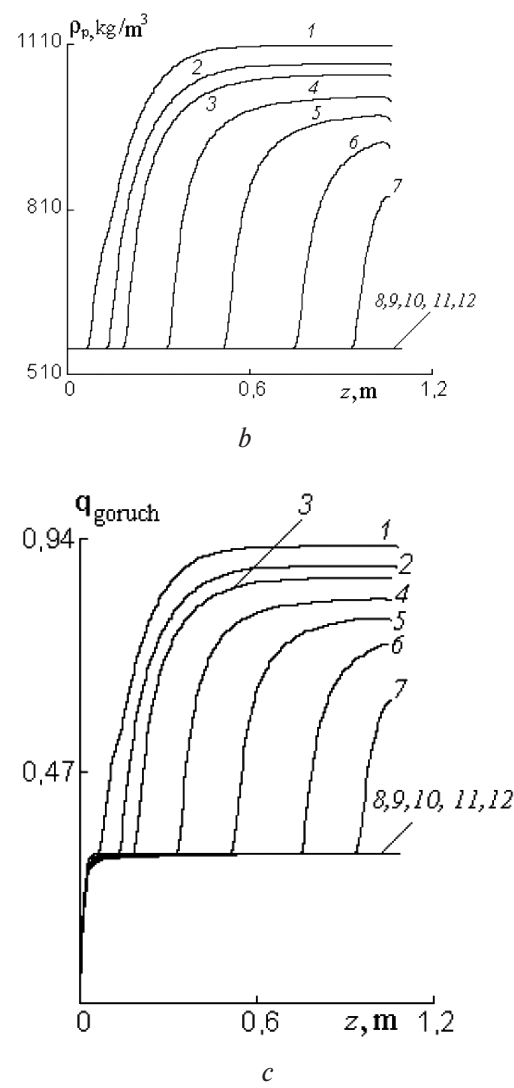

Fig. 6. Distribution of velocities of Volyn peat particles (a), their density (b), combustible matter (c) in the crushed peat through the height of a stationary bed versus time for Option 1:

$1-100 s ; 2-200 s ; 3-300 s ; 4-600 s ; 5-1000 s ; 6-1500 s$; $7-1900 s ; 8-2200 s ; 9-2400 s ; 10-2800 s ; 11-3000 s ;$ $12-3200 s$

bed, motion, heat exchange and chemical reaction of the gasdispersed medium in a steam-air mixture enriched with oxygen under pressure. This information can be used in designing the gas generators, as well as in the implementation of joint combustion technology proposed in this work: in the combustion chamber of TPP-210A boiler, of the polydisperse ensemble of gas coal particles with synthetic peat gas cleared from tar and dust.

With the aid of the constructed model, the process of thermochemical processing of $28939 \mathrm{~kg} / \mathrm{h}$ of raw peat in three reactors with a fixed bed under 1.5 MPa pressure (Option 1) is numerically investigated. The following results are obtained:

a) Composition of generator gas at the outlet of reactors: $R_{\mathrm{CO}}=33.474 \%, \quad R_{\mathrm{H}_{2}}=8.26 \%, \quad R_{\mathrm{H}_{2} \mathrm{O}}=7.82 \%, \quad R_{\mathrm{CO}_{2}}=$ $=3.672 \%, \quad R_{\mathrm{CH}_{4}}=6.94 \%, \quad R_{\mathrm{N}_{2}}=19.81 \%, \quad R_{\mathrm{CH}_{1.193} \mathrm{O}_{0.4083}}=$ $=16.63 \%$ and $R_{\mathrm{C}_{1} \mathrm{H}_{2}}=3.39 \%$ at $\tau=400 \mathrm{~s}$, and $R_{\mathrm{CO}}=52.8 \%$, $R_{\mathrm{H}_{2}}=13.21 \%, R_{\mathrm{N}_{2}}=33.88 \%$ at $\tau=3200$ s. The raw synthet- ic gas in amount of $33005 \mathrm{~kg} / \mathrm{h}$, generated in the stationary gasification process of the Volyn peat particles ( $\tau \geq 2200 \mathrm{~s}$ ), after passing the stage of cleaning from tar and dust, is mixed with $5469.5 \mathrm{~kg} / \mathrm{h}$ of evaporated moisture from the raw fuel and after resetting excess pressure in the gas turbine 34 in the amount of $36720 \mathrm{~kg} / \mathrm{h}$ enters the gas burner of the combustion chamber of TPP-210A boiler. The mass fractions of the components of this gas at the inlet to the furnace are: $\mathrm{H}_{2} \mathrm{O}=0.226$, $\mathrm{H}_{2}=0.00723, \mathrm{CO}=0.405, \mathrm{CO}_{2}=0.0674, \mathrm{CH}_{4}=0.0608, \mathrm{H}_{2} \mathrm{~S}=$ $=0.00472, \mathrm{~N}_{2}=0.229$. This constitutes an initial condition for a joint combustion mode of $36720 \mathrm{~kg} / \mathrm{h}$ synthetic gas ( $20 \%$ by fuel energy) and $48996 \mathrm{~kg} / \mathrm{h}$ of the pulverized gas coal, which will be described in the third part of this work;

b) in Option 1, where zone of maximum heat release is adjacent to the grate, within time interval $2200<\tau<3200 \mathrm{~s}$, a stable (stabilized) stationary process of thermochemical processing of peat is formed with $q_{4,1}=0.13-0.3 \%$;

c) behavior of the distribution of disperse phase velocity resembles a mirror image of the dependence of particle diameters profile. A sharp decrease in the diameter and the speed of ash-coke particles is observed in oxygen, oxidation-reducing zones and in the gasification zone, where intense conversion of ash-coke particles occurs due to heterogeneous chemical reactions.

Analyses of the results of numerical studies on gasification process in the gas generator for Options 2-4 will be given in the second part of this work.

\section{References.}

1. Tiilikka, M., \& Bolhàr-Nordenkampf, M. (2014). Advanced Gasification Technologies for Large Scale Energy Production. Power-Gen Europe Conference, 1, 1-16.

2. Rokhman, B., \& Nekhamin, M. (2020). Theoretical study of nonstationary air gasification of solid fuel in a fixed bed at atmospheric pressure. Vidnovluvana Energetyka, 1(60), 86-95. https://doi. org/10.36296/1819-8058.2020.1(60).86-95.

3. Nagornov, A. N. (2020). Results and development of gasification technologies of low-ash coal in a pressurized dense bed with a steam-air blowing (Abstract of a doctoral thesis of Technical Sciences).

4. Alekseev, M.V., Bogomolov, A.R., Pribaturin, N.A., Shevyrev, S. A., Sorokin, A. L., \& Kagakin, E. I. (2013). Modeling of a layerwise gas counterflow gasifier for conversion of sludge TSOF "Berezovskaya" in a flow of overheated steam. Vestnik Kuzbasskogo gosudarstvennogo universiteta, $79-84$.

5. Taimasov, D. R., Permyakov, E.E., Ulzysayhan, Hudryoe, \& Senachin, P. K. (2017). Coal gasification in the dense bed of the reversed process gas generator. Polzunovsky almanac, 3(1), 24-27.

6. Maryishin, N.S., Timasov, D.R., Permyakov, E.E., \& Senachin, P. K. (2017). Modeling gasification in a dense bed. Energo and resource-efficiency of low-stored buildings. Materials, 338-342.

7. Han, W., \& Gou, X. (2020). Improved path flux analysis mechanism reduction method for high and low temperature oxidation of hydrocarbon fuels. Combustion Theory and Modelling, 24(6), 10901107. https://doi.org/10.1080/13647830.2020.1820577.

8. Nnabuo, N., Salama, A., \& Ibrahim, H. (2020). CFD investigation of biogas reformate using membrane-assisted water gas shift reaction: parametric analyses. Chemical Engineering Research and Design. https://doi.org/10.1016/j.cherd.2020.07.023.

9. Ma, J., \& Zitney, S. E. (2012). CFD modeling of entrained-flow gasifiers with improved physical and chemical submodels. Energy Fuels, 26, 7195-7219.

10. Gómes-Barea, A., \& Leckner, B. (2010). Modeling of biomass gasification in fluidized bed. Progress in Energy and Combustion Science, 36, 449-509.

11. Watanabe, H., \& Kurose, R. (2020). Modeling and simulation of coal gasification on an entrained flow coal gasifier. Advanced Powder Technology. https://doi.org/10.1016/j.apt.2020.05.002.

12. Gerasev, A. P. (2017). Modeling of mass transfer in apparatuses with a stationary granular bed taking into account the phase temperature difference. Theoretical Foundations of Chemical Engineering, 51(3), 320-326. https://doi.org/10.1134/s0040579517030046.

13. Schneiderbauer, S., \& Saeedipour, M. (2018). Approximate deconvolution model for the simulation of turbulent gas-solid flows: An a priori analysis. Physics of Fluids, 30(2), 023301. https://doi. org/10.1063/1.5017004. 
14. Nikolaev, A. K., Dokoukin, V.P., Lykov, Y.V., \& Fetisov, V.G. (2018). Research of processes of heat exchange in horizontal pipeline. IOP Conference Series: Materials Science and Engineering, 327, 032041. https://doi.org/10.1088/1757-899x/327/3/032041.

15. Pinto, R. N., Afzal, A., D’Souza, L. V., Ansari, Z., \& Mohammed Samee, A. D. (2016). Computational Fluid Dynamics in Turbomachinery: A Review of State of the Art. Archives of Computational Methods in Engineering, 24(3), 467-479. https://doi.org/10.1007/s11831016-9175-2.

16. Lü, D., Bai, Y., Wang, J., Song, X., Su, W., Yu, G., ..., Tang, G.-J. (2021). Structural features and combustion reactivity of residual carbon in fine slag from entrained-flow gasification. Journal of Fuel Chemistry and Technology, 49(2), 129-136. https://doi.org/10.1016/ s1872-5813(21)60011-7.

17. Mularski, J., \& Modliński, N. (2020). Entrained flow coal gasification process simulation with the emphasis on empirical devolatilization models optimization procedure. Applied Thermal Engineering, 115401. https://doi.org/10.1016/j.applthermaleng.2020.115401.

\section{Спільне спалювання дрібнодисперсного пилу газового вугілля й синтетичного торф'яного газу. \\ Частина 1. Моделювання процесів пароповітряної газифікації торфу в нерухомому шарі та спалювання пилогазової суміші в потоці}

\section{Б. Б. Рохман, Н.І. Дунаєвська, В.Г. Вифатнюк,} I.B. Безценний

Інститут теплоенергетичних технологій Національної академії наук України, м. Київ, Україна, е-mail: brolene@ yahoo.com
Мета. Побудувати модель газифікації твердого палива у фіксованому шарі з урахуванням швидкості частинок, що дозволяє отримати детальну інформацію щодо технологічного процесу. Розробити оптимальну технологічну схему спільного спалювання в котлі ТПП-210А пиловугільних частинок і торф'яного газу. Дослідити процес спалювання бінарної суміші.

Методика. Об'єктами досліджень були пилоподібне вугілля українських покладів і волинський торф. Для розрахунку процесу газифікації торф'яного газу використовувалась розроблена модель. Для дослідження процесу спалювання бінарної суміші - ANSYS FLUENT.

Результати. Розроблена модель газифікації твердого палива у фіксованому шарі, що відрізняється від існуючих моделей урахуванням швидкості частинок та їі зміни. За її допомогою отримана детальна інформація про температурні й концентраційні поля двофазного середовища. Досліджено процес спалювання бінарної суміші в котлі ТПП-210А.

Наукова новизна. Встановлено, що у часовому інтервалі $2200<\tau<3200$ с формується стійкий стаціонарний процес термохімічної переробки торфу з $q_{4}=0,13-0,3 \%$. Характер розподілу швидкостей дисперсної фази нагадує дзеркальне відображення залежності профілю діаметра частинок.

Практична значимість. За допомогою побудованої моделі чисельно досліджено процес термохімічної переробки 28939 кг/год сирого торфу у трьох реакторах із фіксованим шаром під тиском 1,5 МПа. Визначено склад генераторного газу на виході з реакторів.

Ключові слова: нерухомий шар, вугілля, торф, теплопровідність, пароповітряна газифікація, кондуктивний $i$ радіаційний теплообмін

The manuscript was submitted 10.03.21. 